\title{
A Study of Serum Levels of Vitamin B12, Folic Acid, and Homocysteine in Vitiligo Patients and Controls Attending a Tertiary Care Hospital in Bengaluru - An Analytical Cross-Sectional Study
}

\author{
Priya Kootelu Sundar ${ }^{1}$, Bindu Mohan Chavala², Shanmukha Miyatura Nagaraj ${ }^{3}$, Harshitha Kotla Reddy ${ }^{4}$ \\ 1,3,4 Department of Dermatology, Rajarajeswari Medical College and Hospital, Mysore \\ Road, Bangalore, Karnataka, India. ${ }^{2}$ Department of Biochemistry, Rajarajeswari \\ Medical College and Hospital, Mysore Road, Bangalore, Karnataka, India.
}

\section{ABSTRACT}

\section{BACKGROUND}

Vitiligo is a hypopigmentary disease of the skin which is associated with impaired quality of life and depression. In our present study, we evaluated the serum levels of homocysteine, vitamin B12, and folic acid in patients with vitiligo and controls in a tertiary care centre and also their association with the extent and activity of vitiligo.

\section{METHODS}

An analytical cross-sectional study was conducted on 40 patients with vitiligo and 40 controls visiting a tertiary care centre for a period of one year. Detailed history regarding demographic data, history of disease, family history, duration of the disease etc. was recorded. Vitiligo Disease Activity Score (VIDA SCORE) and vitiligo area scoring index (VASI) of each body region was calculated. After explaining the procedure, fasting ( $5 \mathrm{ml}$ ) blood sample was drawn and the levels of homocysteine, vitamin B12, and folic acid were measured. Data was analysed using SPSS software and $P$ value less than 0.05 was considered statistically significant.

\section{RESULTS}

There was statistical significance between vitiligo patients and controls of serum vitamin $\mathrm{B} 12$, homocysteine and folic acid $(\mathrm{P}<0.05)$ and there was significant association for males in cases and controls $(\mathrm{P}<0.05)$.

\section{CONCLUSIONS}

Vitiligo is associated with elevated levels of serum homocysteine and decreased levels of vitamin B12 and folic acid.

\section{KEY WORDS}

Vitiligo, Homocysteine, Vitamin B12, Folic Acid, Depigmentation
Corresponding Author: Dr. Harshitha Reddy $K$., Department of Dermatology Rajarajeswari Medical College and Hospital, Mysore Road, Bangalore, Karnataka, India. E-mail: reddyharshitha1993@gmail.com

DOI: $10.14260 /$ jemds/2021/464

How to Cite This Article:

Sundar PK, Chavala BM, Nagaraj SM, et al. A study of serum levels of vitamin B12, folic acid, and homocysteine in vitiligo patients and controls attending a tertiary care hospital in Bengaluru - an analytical cross - sectional study. J Evolution Med Dent Sci 2021;10(30):2271-2275,

$10.14260 /$ jemds/2021/464 DOI:

Submission 09-03-2021,

Peer Review 15-05-2021,

Acceptance 21-05-2021,

Published 26-07-2021.

Copyright (C) 2021 Priya Kootelu Sundar et al. This is an open access article distributed under Creative Commons Attribution License [Attribution 4.0 International (CC BY 4.0)] 


\section{BACKGROUND}

Vitiligo being an acquired, multifactorial pigmentary disorder is characterized by depigmentation of the hair follicles and epidermis affecting about $1 \%$ of population overall. The aetiology and the exact mechanism for the destruction of melanocytes in vitiligo has not been fully understood. Many theories like autoimmune, oxidative stress, neurohumoral, and cytotoxic hypothesis have been formulated. ${ }^{1}$

Homocysteine (Hcy) is a highly reactive sulphur containing amino acid that is synthesized during protein catabolism. Folic acid and vitamin B12 serve as cofactors of homocysteine methyltransferase for the conversion of homocysteine methionine to methionine and it is metabolized by: transsulfuration, remethylation and depending on folate and vitamin B12 levels. Hence, any nutritional deficiency in one of these two vitamins will lead to an increase in Hcy levels in the circulation and results in hyperhomocysteinaemia and levels of these three compounds appear to be inversely interconnected which leads to variety of diseases. ${ }^{2}$ Earlier literature had reported that hyper homocystinaemia disrupts melanogenesis via various mechanisms, such as increased oxidative stress, through activation of various cytokines, and tyrosinase activity inhibition. ${ }^{3}$ Studies have shown that supplementation with folic acid and vitamin B12 causes repigmentation of vitiligo lesions. ${ }^{4}$

Methylenetetrahydrofolate reductase (MTHFR) is a vital regulatory enzyme involved in the conversion of homocysteine to methionine which catalyses the reduction of 5, 10-methylenetetrahydrofolate to 5 - methyltetrahydrofolate and its gene being located on chromo some $1 \mathrm{p} 36.3$ and consists of 11 exons and 10 introns. Polymorphisms of MTHFR i.e., $677 \mathrm{C}>\mathrm{T}$ and $1298 \mathrm{~A}>\mathrm{C}$ result in reduced activity of MTHFR enzyme and affect homocysteine levels. These two polymorphisms of MTHFR have been reported to be associated with several diseases including vitiligo. ${ }^{5}$ Excess Hcy has been shown to induce oxidative stress which is associated with a wide cellular destruction in vascular smooth muscle cells and it is also associated with a wide variety of medical conditions such as systemic lupus erythematosus and psoriasis. It is reported that homocystinuria is associated with fair skin and hair, a phenomenon often described as pigmentary dilution. ${ }^{6}$

Recently a study conducted in Egypt. ${ }^{7}$ showed that serum Hcy is elevated in vitiligo patients when compared with healthy subjects. Reactive oxygen species which are produced due to oxidation of hcy leads to oxidative stress on melanocytes. Tyrosinase is a $75 \mathrm{kD}$ copper-containing enzyme that starts the melanin biosynthesis in pigmented cells. Binding of Hcy with copper leads to inhibition of tyrosinase enzyme at its active site resulting in reversible hypopigmentation. ${ }^{8}$ Similarly Singh et al. ${ }^{9}$ reported that serum homocysteine level was significantly increased while folic acid and Vitamin B12 levels were significantly decreased in vitiligo group as compared to controls. On the contrary, in another study conducted by Balci et al.10 no association was found between Hcy, Vitamin B12, folic acid and vitiligo.

From the above studies it can be seen that the data on the serum levels of vitamin B12, homocysteine and folic acid in patients with vitiligo are mostly conflicting and there have been controversies over the role of these factors in the pathogenesis of vitiligo.

With this background the present study intended to evaluate Hcy, folic acid, and vitamin B12 serum levels in vitiligo patients and controls and study the importance of these levels in the pathogenesis of vitiligo and their relation to vitiligo activity and extent.

\section{METHODS}

It is an analytical cross-sectional study which included patients attending the outpatient department of Department of Dermatology in Rajarajeswari medical college and hospital, Bengaluru. The study period was between January 2019 and January 2020

\section{Sample Size and Sampling Technique}

Our study included 40 vitiligo patients and 40 age \& sex matched controls on the basis of other Indian studies on vitiligo and the average number of vitiligo cases attending the OPD of RRMCH in the last three years.

\section{Inclusion Criteria}

- The age of the patients ranged from 18 to 50 years.

- Vitiligo patients diagnosed clinically using VADI and VASI scores were included.

\section{Exclusion Criteria}

- Pregnant women

- History of smoking, alcohol intake

- Intake of Vitamins \& hormonal therapy,

- Anaemia

- Diseases which are known to alter the Hcy level such as genetic disorders of amino acid metabolism, hypertension, cardiovascular disease, diabetes mellitus, thyroid dysfunction, renal failure, deep venous thrombosis, Behcet's disease, and psoriasis.

\section{Ethical and Informed Consent}

After getting the approval from Institutional ethical committee, informed consent was taken from the cases and controls before starting the study.

\section{Data Collection}

Detailed history regarding demographic data, history of disease, family history, duration of the disease, progression and distribution of the lesions and clinical type was recorded. Active or progressive vitiligo and stable disease assessment was carried out by evaluating the activity of the disease according to the Koebner phenomenon and the history of new lesions in the last one year. Vitiligo Disease Activity Score (VIDA score) was obtained on the basis of opinion of the individual patient regarding the presence of disease activity over time. 


\begin{tabular}{|cc|}
\hline VIDA Score & Activity of Disease \\
+4 & Activity of 6 weeks or less duration \\
+3 & Activity of 6 weeks to 3 months \\
+2 & Activity of $3-6$ months \\
+1 & Activity of $6-12$ months \\
0 & Stable for 1 year or more \\
\hline-1 & Stable with spontaneous repigmentation since 1 yr. or more \\
\hline & Table 1. VIDA Score \\
\hline
\end{tabular}

\section{VASI Score Calculated for Each Patient}

The percentage of vitiligo involvement was calculated in terms of hand units. One hand unit (which encompasses the palm plus the volar surface of all digits) is approximately equivalent to $1 \%$ of the total body surface area. The degree of pigmentation was estimated as: $100 \%$ - complete depigmentation, no pigment; $90 \%$ - specks of pigment; $75 \%$ de pigmented area which exceeds the pigmented area; $50 \%$ pigmented and depigmented areas are equal; $25 \%$ pigmented area which exceeds depigmented area; and $10 \%-$ only specks of depigmentation. The VASI for each body area was calculated by the product of the area of vitiligo in hand units and the extent of depigmentation within each hand unit measured patch. ${ }^{11}$ After explaining the procedure, fasting (5 ml) blood sample was drawn under proper aseptic precautions and added to vacutainers. The blood was allowed to clot for 10 mins, centrifuged at $3000 \mathrm{rpm}$ and serum was collected. Serum was stored at $-20^{\circ}$ C \& homocysteine, vitamin B12 and folic acid levels were measured. Serum homocysteine levels were assessed using Accurex Biochemical Private Ltd kits on Erba EM 360 fully automated clinical chemistry analyser. Normal Serum Homocysteine value taken was $<15 \mathrm{mmol} /$ L. Levels of Vitamin B12 and serum folic acid were quantitatively determined using competitive ligand labelled protein binding immunoluminometric assay by fully automated chemiluminescence immunoassay (CLIA) analyser Malgumi 1000. The normal ranges for them were $200-1100 \mathrm{pg}$ / $\mathrm{ml}$ and 5.21 - $20 \mathrm{ng} / \mathrm{ml}$ respectively.

\section{Statistical Analysis}

Serum levels (serum homocysteine, serum vitamin B12 and serum folic acid) were assessed as primary outcome variables. Study groups (cases and controls) were assessed as primary explanatory variables. Demographic parameters like (age, gender, dietary history, duration, disease activity, classification and specific biochemical were considered as other study relevant variables.
All quantitative variables were analysed for normal distribution within each group of explanatory variables by using histograms and normality $Q$ - $Q$ plots. Shapiro-Wilk test was done to assess normal distribution. Shapiro-Wilk test $P$ value of more than 0.05 was considered as normal distribution. For normally distributed quantitative parameters the mean values were compared between study groups using independent sample $\mathrm{t}$ - test (cases and controls). Association between quantitative explanatory and outcome variables was assessed by calculating Spearman's rank correlation coefficient.

Outcomes of categorical data were compared between study groups using chi square test or Fisher's Exact test (If the overall sample size was $<20$ or if the expected number in any one of the cells was $<5$, Fisher's exact test was used). P value less than 0.05 was considered statistically significant. IBM SPSS version 22 was used for statistical analysis.

\section{RESULTS}

A total of 80 subjects were included in the final analysis. The difference in dietary history between the cases and controls was found to be insignificant with a $\mathrm{P}$ - value of 0.499 , with majority of $24(60 \%)$ participants in cases. Among cases, 15 (37.5 \%) had stable disease activity and 25 (62.5\%) participants had unstable disease activity. Among localized cases, 15 were focal, 6 segmental, 4 mucosal and 15 (37.5\%) participants were generalized. There was statistically significant difference between specific biochemical parameters like, serum homocysteine, serum vitamin B12 and serum folic acid $(\mathrm{P}<0.05)$. There was statistical significance between cases and controls of males in serum levels of homocysteine, serum vitamin B12 and serum folic acid $(\mathrm{P}<$ 0.05).

The mean difference of serum homocysteine between cases and controls of females was statistically not significant ( $P$ value 0.414 ) and the mean difference of vitamin B12 and folic acid between cases and controls of females was statistically significant with $\mathrm{P}$ value of 0.004 and 0.016 respectively. There was no statistical significance between disease activity and serum levels of vitamin B12, homocysteine and folic acid $(\mathrm{P}>0.05)$. There was no statistical significance between duration and serum levels of homocysteine, vitamin B12 and folic acid.

\begin{tabular}{|c|c|c|c|c|}
\hline \multirow{3}{*}{ Dietary History } & Veg & $16(40 \%)$ & $19(47.5 \%)$ & \multirow{2}{*}{0.499} \\
\hline & Non - veg & $24(60 \%)$ & $21(52.5 \%)$ & \\
\hline & Duration(Mean $\pm S D$ ) & $8.36 \pm 1.61$ & 0 & $*$ \\
\hline \multirow{2}{*}{ Disease Activity } & Stable & $15(37.5 \%)$ & $0(0 \%)$ & \multirow[t]{2}{*}{ * } \\
\hline & Unstable & $25(62.5 \%)$ & $0(0 \%)$ & \\
\hline \multirow{8}{*}{ Classification } & Localized & $25(62.5 \%)$ & $0(0 \%)$ & \multirow{8}{*}{ * } \\
\hline & Focal & $15(60 \%)$ & $0(0 \%)$ & \\
\hline & Mucosal & $4(16 \%)$ & $0(0 \%)$ & \\
\hline & Segmental & $6(24 \%)$ & $0(0 \%)$ & \\
\hline & Generalized & $15(37.5 \%)$ & $0(0 \%)$ & \\
\hline & Vulgaris & $9(60 \%)$ & $0(0 \%)$ & \\
\hline & Acrofacial & $2(13.33 \%)$ & $0(0 \%)$ & \\
\hline & Mixed & $4(26.67 \%)$ & $0(0 \%)$ & \\
\hline \multirow{3}{*}{ Specific biochemical (Mean \pm SD) } & Serum Homocysteine (< 15 mmol / L.) & $14.97 \pm 6.77$ & $12.16 \pm 5.29$ & 0.042 \\
\hline & Serum Vitamin B12 (200 - $1100 \mathrm{pg} / \mathrm{ml})$. & $227.32 \pm 45.08$ & $280.44 \pm 74.53$ & $<0.001$ \\
\hline & Serum Folic Acid 5.21 - $20 \mathrm{ng} / \mathrm{ml}$.) & $5.63 \pm 2.11$ & $7.2 \pm 2.43$ & 0.003 \\
\hline \multicolumn{5}{|c|}{ Table 2. Comparison of Base Line Parameters in Study Group $(N=40)$} \\
\hline
\end{tabular}




\begin{tabular}{|c|c|c|c|c|c|c|}
\hline \multirow{3}{*}{ Serum Levels } & \multicolumn{5}{|c|}{ Gender $($ Mean \pm SD) } & \multirow{3}{*}{ P Value } \\
\hline & \multicolumn{2}{|c|}{ Male } & \multirow{2}{*}{ P Value } & \multicolumn{2}{|c|}{ Female } & \\
\hline & Cases & Controls & & Cases & Controls & \\
\hline Homocysteine ( $<15 \mathrm{mmol} / \mathrm{L}$.) & $14.91 \pm 5.57$ & $10.62 \pm 5.32$ & 0.02 & $15.01 \pm 7.66$ & $13.41 \pm 5.03$ & 0.414 \\
\hline Vitamin B12 (200 - 1100pg / ml.) & $230.76 \pm 44.79$ & $287.48 \pm 87.77$ & 0.02 & $224.78 \pm 46.12$ & $274.68 \pm 63.26$ & 0.004 \\
\hline Folic Acid (5.21 - $20 \mathrm{ng} / \mathrm{ml}$ ) & $5.84 \pm 1.74$ & $7.21 \pm 2.69$ & 0.08 & $5.48 \pm 2.37$ & $7.20 \pm 2.25$ & 0.016 \\
\hline \multicolumn{7}{|c|}{ Table 3. Comparison of Serum Levels and Gender between Study Groups $(N=80)$} \\
\hline
\end{tabular}

\begin{tabular}{|cccc|}
\hline Parameter (Mean \pm SD) & \multicolumn{2}{c}{ Disease Activity } & P Value \\
Stable & Unstable & \\
Serum Homocysteine $(<15 \mathrm{mmol} / \mathrm{L})$. & $13.28 \pm 7.23$ & $15.98 \pm 6.41$ & 0.243 \\
Serum Vitamin B12 (200 - 1100pg / ml.) & $228.44 \pm 51.03$ & $226.65 \pm 42.21$ & 0.905 \\
Serum Folic Acid (5.21 - 20 ng / ml.) & $6.23 \pm 2.09$ & $5.27 \pm 2.07$ & 0.165 \\
\hline Table 4. Comparison of Serum Levels between & \\
Disease Activity in the Study Population $(\mathbf{N}=\mathbf{8 0})$ & \\
\hline
\end{tabular}

\begin{tabular}{|ccc|}
\hline Parameter & Age & Duration \\
\hline Serum Homocysteine & -0.214 & 0.098 \\
P value & 0.184 & 0.547 \\
Serum Vitamin B12 & -0.065 & -0.029 \\
P value & 0.690 & 0.859 \\
Serum Folic Acid & -0.13 & -0.134 \\
P Value & 0.423 & 0.409 \\
\hline Table 5. Spearman Rank Correlation between Serum Levels with \\
Patient Profile in the Study Population $(\mathbf{N}=\mathbf{8 0})$ \\
\hline
\end{tabular}

\section{DISCUSSION}

The present study showed statistical significance between vitiligo patients and controls for serum vitamin B12, homocysteine and folic acid levels $(\mathrm{P}<0.05)$. The association was stronger for males between cases and controls $(\mathrm{P}<0.05)$. The disease was stable in 15 (37.5\%) participants, among which 25 (62.5\%) participants had localised vitiligo, 15 (37.5 $\%)$ participants had generalised vitiligo. These findings were in agreement with Arpaia et al. $^{12}$ where the disease was stable in 20 and progressive in 28 patients, and twelve patients had localised and 34 had generalised, while the remaining two were universal - type.

The mean serum Hcy levels ( $<15 \mathrm{mmol} / \mathrm{L}$.) were higher in vitiligo cases $14.97 \pm 6.77$ when compared to controls 12.16 \pm 5.29 which was statistically significant $(P-0.042)$, this finding was similar to a study done by Gupta, et al. ${ }^{13}$ where mean serum Hcy levels were found to be higher than reference standards in both cases and controls. This may be justified partly on the basis of distinct Indian dietary habits and also by genetic polymorphisms. It has been observed that most Indians adhere to a vegetarian diet and the non-vegetarian ones consume a lesser amount of animal - derived protein than their Western counterparts and cobalamin was found deficient in Indian diet which itself may lead to increased Hcy. In the absence of a significant alteration in the levels coupled with a lack of correlation with any of the disease characteristics, this study by Gupta, et al. ${ }^{13}$ could not substantiate the role of Hcy in vitiligo. In addition, suction induced blister fluid analysis of active vitiligo patients was also reported to have elevated homocysteine levels in a study conducted by Anbar et al. ${ }^{6}$ The finding was in contrast to a study reported by Zaki et al.14 where there was no significant difference between serum homocysteine in vitiligo and control $(\mathrm{P}=0.905)$.

In the present study, the mean value of serum homocysteine was higher and serum vitamin B12 and folic acid levels were noted to be lower ( $P$ - 0.001) in vitiligo patients as compared to controls. Both vitamin B12 and folic acid are required as cofactors by the enzyme homocysteine methyl transferase for the regeneration of methionine from homocysteine in the methyl cycle. And nutritional deficiency in one of these two vitamins will result in an increase in homocysteine and a decrease in methionine levels in the circulation.

Homocysteine levels are determined by serum levels of Vitamin B12 and folic acid. The results obtained were in accordance with a recent Indian study done by Agarwal et al.15 who reported that the mean values of Vitamin B12 and folate were significantly decreased in vitiligo group as compared to controls. In this study we reported VASI score qualitatively. This is in contrast to a study by Hasibuan et al. ${ }^{16}$ where they did not find any significant correlation between VASI score and serum homocysteine level $(\mathrm{P}>0.05 ; \mathrm{r}=0.281)$.

The microenvironment of skin in patients affected with vitiligo is already compromised due to many factors such as increased proinflammatory cytokines, altered miRNA expression, which in turn makes the melanocytes vulnerable. Earlier it was speculated that initial triggering event to precipitate vitiligo was oxidative stress and exacerbated by contributing autoimmune factors along with oxidative stress, and endoplasmic reticulum stress which is known to be caused due to homocysteine could be a potential link between oxidative stress and autoimmunity in vitiligo and the ability of homocysteine to induce oxidative stress, ER stress, inflammatory and immune-modulatory mechanisms suggest that homocysteine might be playing an important role as initial triggering factor and also in the progression of vitiligo. ${ }^{17}$

One of the advantages of using homocysteine and vitamin B12 as activity markers is that they are easy to measure and also contributes to evidence based medicine. However, the etiopathogenesis of vitiligo is very complicated as it involves multiple pathways and also is difficult to find a single biomarker which can suggest every aspect of the underlying mechanisms. ${ }^{18}$

\section{CONCLUSIONS}

Elevation of serum Hcy level and lower vitamin B12 and folic acid levels might be aggravating factors for vitiligo in predisposed individuals and that alteration of serum Hcy appears to be related to the sex of the patient and the activity of the disease. Future research on vitiligo is needed to determine and explore the therapeutic potential of homocysteine - lowering strategies.

\section{Limitations}

The limitation of this study was fewer number of patients, since this study was only a preliminary study to investigate the relationship between serum homocysteine levels and vitiligo. Hence we suggest further studies to confirm these preliminary results with importance given on therapeutic trials which can help in the assessment of elevated Hcy levels, depending on the prognosis of vitiligo. Another limitation was that, serum levels of homocysteine, folic acid, and vitamin B12 can be 
influenced by various factors, such as the baseline nutritional status, diet and comorbidities. Further research on vitiligo is necessary to confirm the underlying mechanisms of hyperhomocystinaemia, vitamin B12 and folic acid deficiency which should include a large sample size and broader geographical area using well designed Cohort studies.

Data sharing statement provided by the authors is available with the full text of this article at jemds.com.

Financial or other competing interests: None.

Disclosure forms provided by the authors are available with the full text of this article at jemds.com.

\section{REFERENCES}

[1] Ezzedine K, Eleftheriadou V, Whitton M, et al. Vitiligo. Lancet 2015;386(9988):74-84.

[2] El-Dawela RE, Abou-Elfetouh S. Relationship between homocysteine, vitamin B12, folic acid levels and vitiligo. J Appl Sci Res 2012;8(11):5528-35.

[3] Kim J, Kim H, Roh $H$, et al. Causes of hyperhomocysteinemia and its pathological significance. Arch Pharm Res 2018;41(4):372-83.

[4] Kumar A, Palfrey HA, Pathak R, et al. The metabolism and significance of homocysteine in nutrition and health. Nutr Metab (Lond) 2017;14(1):78.

[5] Chen JX, Shi Q, Wang XW, et al. Genetic polymorphisms in the methylenetetrahydrofolate reductase gene (MTHFR) and risk of vitiligo in Han Chinese populations: a genotype-phenotype correlation study. Br J Dermatol 2014;170(5):1092-9.

[6] Anbar T, Zuel-Fakkar NM, Matta MF, et al. Elevated homocysteine levels in suction-induced blister fluid of active vitiligo lesions. Eur J Dermatol 2016;26(1):64-7.

[7] Shaker OG, El-Tahlawi SMR. Is there a relationship between homocysteine and vitiligo? A pilot study. Br J Dermatol 2008;159(3):720-4.
[8] Singh S, Singh U, Pandey SS. Increased level of serum homocysteine in vitiligo. J Clin Lab Anal 2011;25(2):1102.

[9] Singh S, Singh U, Pandey S. Serum folic acid, vitamin B12 and homocysteine levels in Indian vitiligo patients. Egypt Dermatol Online J 2012;8(1):1-7.

[10] Sabry HH, Sabry JH, Hashim HM. Serum levels of homocysteine, vitamin B12, and folic acid in vitiligo. Egypt J Dermatol Venerol 2014;34(1):65.

[11] Maryam G, Vahide L, Abbas F. Serum levels of vitamin B12, folic acid and homocysteine in patients with vitiligo. Iran J Dermatol 2015;18(2):45-50.

[12] Arpaia N, Filotico R, Mastrandrea V, et al. Acral viral wart showing a parallel ridge pattern on dermatoscopy. Eur J Dermatol 2009;19(4):381-2.

[13] Gupta S, D'Souza P, Dhali T, et al. Serum homocysteine and total antioxidant status in vitiligo: a case control study in Indian population. Indian J Dermatol 2016;61(2):131-6.

[14] Zaki AM, Abdo HM, Ibrahim IM, et al. Serum homocysteine and vitiligo. The Gulf Journal of Dermatology and Venereology 2014;21(2):15-20.

[15] Agarwal S, Mendiratta V, Chander R, et al. Study of serum levels of vitamin B12, folic acid and homocysteine in vitiligo. Pigment International 2015;2(2):76-80.

[16] Hasibuan DRU, Putra IB, Jusuf NK. Correlation between serum homocysteine and vitiligo area scoring index. Maced J Med Sci 2017;5(3):332-4.

[17] Jadeja SD, Mansuri MS, Singh M, et al. Association of elevated homocysteine levels and methylenetetrahydrofolate reductase (MTHFR) 1298 A > C polymorphism with Vitiligo susceptibility in Gujarat. J Dermatol Sci 2018;90(2):112-22.

[18] Tsai TY, Kuo CY, Huang YC. Serum homocysteine, folate and vitamin B12 levels in patients with vitiligo and their potential roles as disease activity biomarkers: a systematic review and meta-analysis. J Am Acad Dermatol 2019;80(3):646-54.e5. 